\title{
Dual-incision laparoscopic spleen-preserving distal pancreatectomy
}

\author{
Eun Young Kim, Young Kyoung You, Dong Goo Kim, Soo Ho Lee, Jae Hyun Han, Sung Kyun Park, Gun Hyung \\ $\mathrm{Na}$, Tae Ho Hong \\ Department of Surgery, Seoul St. Mary's Hospital, The Catholic University of Korea, Seoul, Korea
}

\begin{abstract}
Laparoscopic spleen-preserving distal pancreatectomy has been widely performed for benign and borderline malignancy in the body or tail of the pancreas when there are not oncologic indications for splenectomy. As the need for minimally invasive procedures to reduce postoperative morbidity and improve the quality of life is increasing, many surgeons have attempted to reduce the number of trocars and incision size to minimize access trauma and scarring. Single-port laparoscopic spleen-preserving distal pancreatectomy is the result of these efforts; however it has many limitations such as technical difficulty and prolonged operation time. In this article, we report the first case of dual-incision laparoscopic spleen-preserving distal pancreatectomy, proving that it can be a safe and feasible minimally invasive procedure for benign or borderline malignant tumors in the body or tail of the pancreas.
\end{abstract}

[Ann Surg Treat Res 2015;88(3):174-177]

Key Words: Dual incision, Spleen-preserving distal pancreatectomy, Laparoscopy

\section{INTRODUCTION}

Laparoscopic distal pancreatectomy (LDP) is a commonly performed surgery for benign and borderline malignancies in the body or tail of the pancreas [1]. In the past, LDP was generally performed with splenectomy. However since the spleen has been found to have an important immunologic role and splenectomy can lead to severe complications such as overwhelming postsplenectomy sepsis, subphrenic abscess or hematological abnormalities such postoperative thrombocytosis [2,3], laparoscopic spleen-preserving distal pancreatectomy (LSPDP) is considered preferable unless there are any oncologic indications for splenectomy.

Single-port laparoscopic surgery is being performed more often as surgical skills and laparoscopic instruments are improved and the demands for minimally invasive procedures increase. However, single-port LSPDP has many limitations including technical difficulty and prolonged operation time. Here we introduce dual-incision laparoscopic spleen-preserving distal pancreatectomy (DILSPDP), which is a minimally invasive procedure that is expected to reduce access trauma compared to conventional LSPDP.

\section{SURGICAL TECHNIQUE}

A 32-year-old man without a history of abdominal discomfort was admitted to the Seoul St. Mary's Hospital with a pancreatic tail mass found on CT scan. He had no significant medical history or prior abdominal surgery, and the mass was incidentally discovered during a health screening. The physical examination was not contributory and clinical laboratory findings including tumors marker (CA 19-9) were normal. The
Received January 27, 2014, Reviewed February 3, 2014,

Accepted February 12, 2014

\section{Corresponding Author: Tae Ho Hong}

Department of Surgery, Seoul St. Mary's Hospital, The Catholic University of Korea College of Medicine, 222 Banpo-daero, Seocho-gu, Seoul 137-

701, Korea

Tel: +82-2-2258-2876, Fax: +82-2-595-2822

E-mail: gshth@catholic.ac.kr

\section{Copyright (c) 2015, the Korean Surgical Society}

(c) Annals of Surgical Treatment and Research is an Open Access Journal. All articles are distributed under the terms of the Creative Commons Attribution NonCommercial License (http://creativecommons.org/licenses/by-nc/3.0/) which permits unrestricted non-commercial use, distribution, and reproduction in any medium, provided the original work is properly cited. 

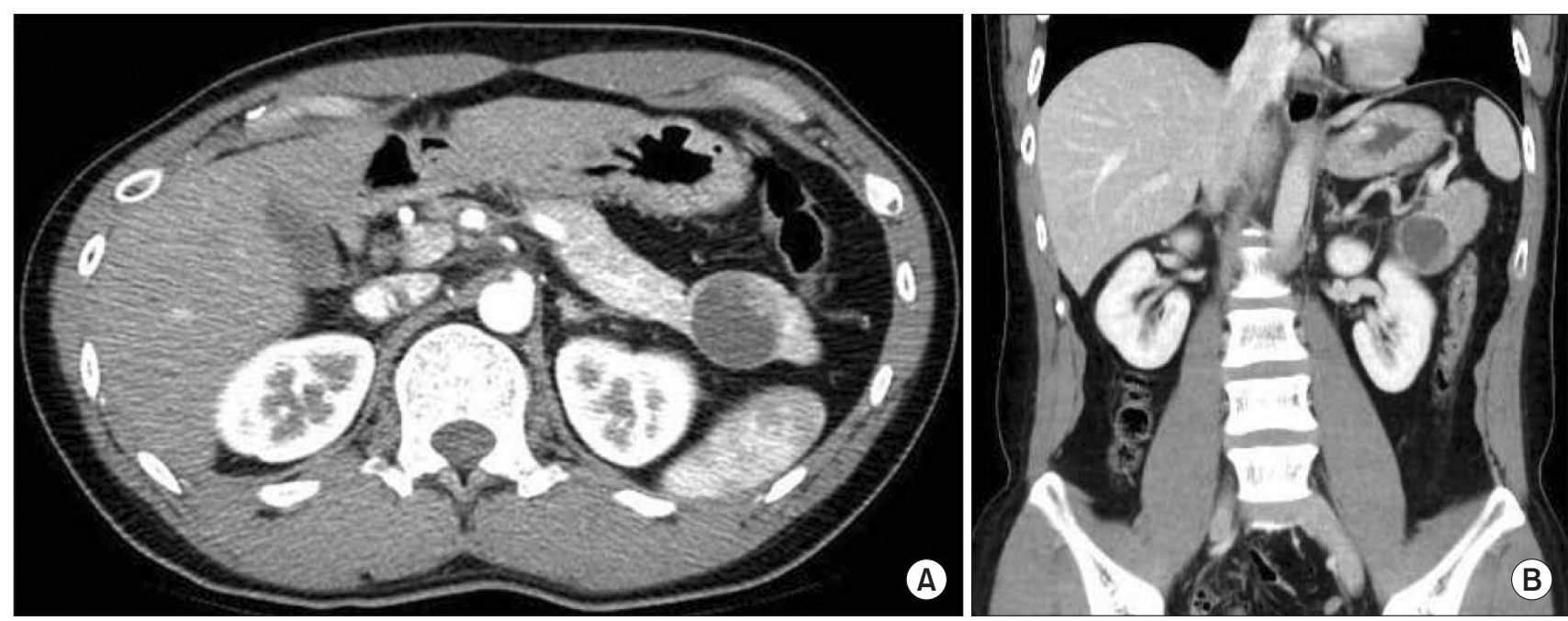

Fig. 1. A $3.3 \mathrm{~cm} \times 3.0 \mathrm{~cm}$ well-defined cystic mass in the tail of the pancreas. This mass has a peripheral solid portion with strong enhancement. (A) Axial view, (B) Coronary view.

computed tomography scan revealed a single, well-defined mixed cystic and solid mass $(3.3 \mathrm{~cm} \times 3.0 \mathrm{~cm})$ that was located in the tail of the pancreas. This mass was did not involve the main pancreatic duct and there was no abnormal lymph node enlargement or vessel invasion (Fig. 1). The patient was informed about the surgical procedure and we obtained his consent for the operation.

Under general anesthesia, the patient was placed in the right lateral decubitus position in a reverse Trendelenburg position and then bent at the waist to increase the space between the subcostal margin and the iliac crest. We made a 3-cm transverse incision at the midclavicular line in the left midabdominal quadrant, and this was extended down to the layer of the abdominal fascia. We used a multichannel trocar (Glove port, Nelis, Seoul, Korea) (Fig. 2), which was composed of four trocars and two rings with gas insulation and exsufflation gates. Specific details about this specially designed trocar can be found in our previous report [4]. This trocar provided access for a 10-mm 30-degree, rigid laparoscope and a 12-mm endovascular stapler (endoscopic linear stapler) and 5-mm laparoscopic instruments. Use of this specialized multichannel trocar enabled the operator to manipulate several instruments with (his or her) right hand simultaneously. A 5-mm laparoscopic grasper was used to displace the spleen to avoid injuring it during dissection and the pancreas was transected using the 12-mm endovascular stapler. The $10-\mathrm{mm}$ rigid laparoscope provided a magnified view of the surgical field, and delicate dissection of the peripancreatic tissue was possible by using the laparoscopic dissector and shears.

An additional 5-mm trocar was placed in the subxiphoid area and used as the access point for an additional 5-mm laparoscopic grasper or dissector; it served as the working

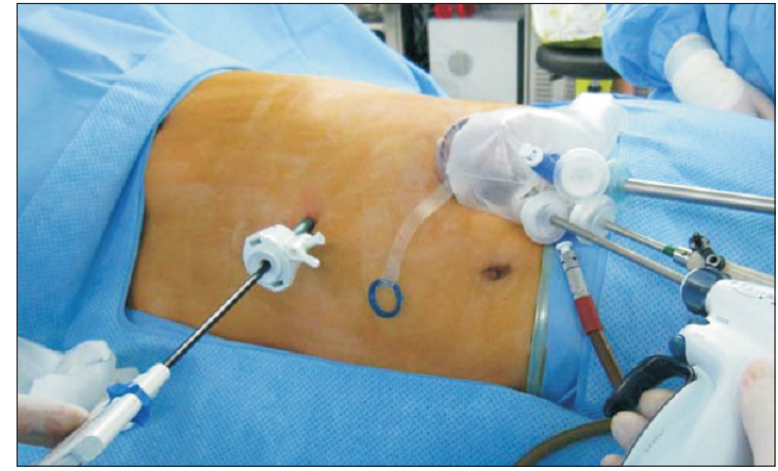

Fig. 2. Extracorporeal view of the dual-incision laparoscopic spleen preserving distal pancreatectomy. We used a multichannel trocar (Glove port, Nelis, Seoul, Korea), which was composed of four trocars and two rings with gas insulation and exsufflation gates.

channel for the operator's left hand. This method created a more ergonomic environment, enabling the operator to use instruments with both of his both hands harmoniously. This technique also was useful when lifting the stomach and pulling the right side of the pancreas downward (Fig. 3).

After inserting the trocars, the splenocolic ligament was divided and after the splenic flexure of the colon was displaced caudally, the gastrocolic ligaments were divided to assess the lesser sac. The lesser sac was entered using ultrasonic coagulating shears (Harmonic ACE scalpel, Ethicon Inc., a division of Johnson \& Johnson, Cincinnati, $\mathrm{OH}$, USA) to divide the omentum along the greater curvature of the stomach, and the anterior surface of the body and tail of the pancreas were exposed. The lateral incision was extended to the level of the short gastric vessels and injury to the short gastric vessels 


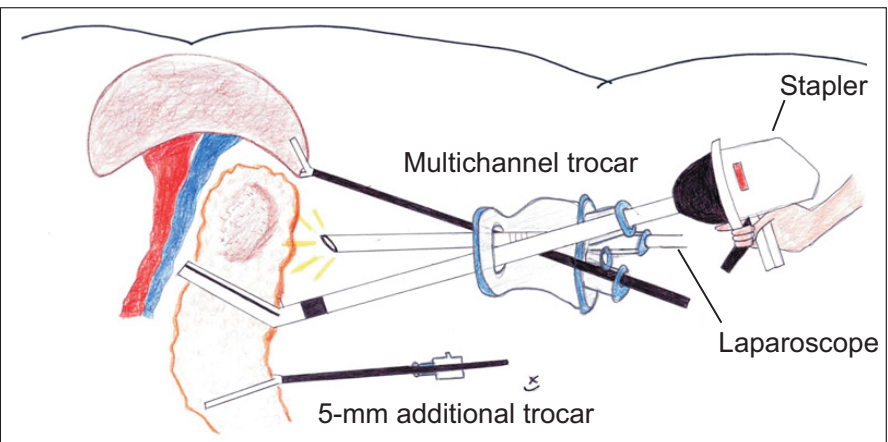

Right lateral decubitus position

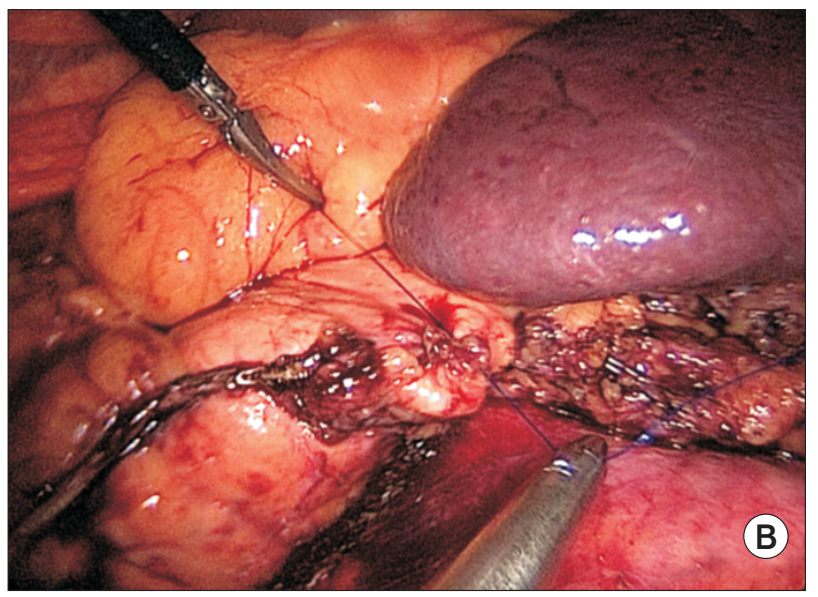

Fig. 3. Schematic diagram and intracorporeal view of the operation. (A) The operator uses his right hand with several instruments including laparoscopic dissector or grasper, and 12-mm endovascular stapler with 10-mm rigid laparoscope. (B) In the ergonomics of dual-incision laparoscopic spleen-preserving distal pancreatectomy, the operator also could use his left hand with an additional laparoscopic instrument via 5-mm subxiphoid trocar. It makes intracorporeal sutures easy.

was avoided. After the adhesion between the posterior gastric wall and surface of the pancreas was released, dissection was performed along the posterior surface of the pancreas within an avascular line, and then the inferior border of the pancreas and the posterior aspect of the pancreas were mobilized. The splenic vessels were identified at the upper border of the pancreas, and the splenic artery and vein were gently dissected and freed from the pancreas. Small venous branches of splenic vessels that drain the tail of pancreas were divided using a vesselsealing device (LigaSure V, Tyco Healthcare, Tokyo, Japan) and the distal pancreas was fully mobilized. Pancreas transection was performed using an endoscopic linear stapler (Echelon Flex 60 Endopath Stapler with white cartilage, Ethicon Inc.), allowing an adequate margin from the mass (about $1 \mathrm{~cm}$ ).

After transection, the stump of the resected pancreas was lifted anteriorly and the dissection was continued toward the tail until the resected pancreas was released. The resection margin of the remnant pancreas was repaired by laparoscopic running-suturing using prolene \#4-0, and fibrin glue was applied to the stump. One closed suction drain was placed in the pancreas stump through the subxiphoid port site. The specimen was bagged and retrieved through the $3-\mathrm{cm}$ transverse incision after removing the multichannel trocar without extending the incision.

The operating time was 143 minutes and the estimated blood loss was $50 \mathrm{~mL}$. The postoperative course was uneventful and the patient started oral intake on postoperative day 5 . The drain was removed and the patient was discharged on postoperative day 7. The final pathologic diagnosis was solid pseudopapillary epithelial neoplasm.

\section{DISCUSSION}

LDP has been widely accepted as a standard procedure for benign and borderline malignancy in the body or tail of the pancreas [5]. Recently, as the immunological role of the spleen has been more emphasized and many severe complications have been reported after splenectomy [6], LSPDP has been more commonly performed when there are no oncologic indications for splenectomy.

The utility of minimally invasive surgery has been proven by previous studies reporting lesser pain, shorter hospital stays, improved cosmetic outcome and better quality-of-life after surgery [7]. Single-port surgery is one such minimally invasive procedures performed for many surgical purposes including appendectomy [8], hernia repair and cholecystectomy [9]. However, only a few surgeons use minimally invasive techniques for pancreatectomy [10]. Single-port LSPDP can minimize the surgical invasiveness, but it requires more advanced skills and instruments, prolonged operation time, and highly experienced surgeons [7], and these limitations make surgeons hesitant to employ this technique.

In this case, we performed DILSPDP using a multichannel trocar that provided simultaneous access to the abdominal cavity for a laparoscope and 5-mm instruments, and we also used an additional subxiphoid 5-mm trocar as a working port for the operator's left hand. This procedure enables the operator to manipulate instruments in both hands to allow for fine movements. Given that it provides a comfortable environment similar to that in conventional LDP with four ports, the operation time could be reduced to that of conventional LSPDP, and it in our case the operation time was faster than that of single-port LSPDP. In addition to the 
operation time, the technical advantages of DILSPDP provide a safe and effective way to control bleeding or leakage at the cut surface of the pancreas by allowing for intracorporeal suturing. Because a pancreatic fistula can occur during stapling, which carries the risk of considerable bleeding from the stapler line, reinforcement suturing is important, especially in the case of resection of a bulky area such as the body of the pancreas. In single-port LSPDP, insufficient suturing is possible because of technically difficulties; however, DILSPDP enables the operator to adapt to this environment effectively because the favorable ergonomics allows easy laparoscopic intracorporeal suturing. Moreover the operator does not need to adapt to new equipment or unfamiliar movements of the laparoscopic instruments in DILSPDP, therefore the surgeon who performs conventional LSPDP could adapt to the DILSPDP without a long learning curve.

In conventional LSPDP, the incision needs to be elongated to accommodate the length of the specimen during extraction because retrieval of an intact pancreatic specimen is essential for accurate pathologic analysis. In DILSPDP there is no need to elongate the incision, and the specimen can be retrieved through the multichannel trocar site without any additional procedures. In addition, DILSPDP has benefit in terms of operative costs compared to conventional LSPDP because DILSPDP uses a multichannel trocar whereas conventional LSPDP requires four or five trocars. Additionally, reducing the number of trocars reduces the risk of access trauma during trocar insertion. Although we cannot compare the actual results due to limited data, we expect that DILSPDP has the many advantages of minimally invasive surgery including less bleeding and pain. An objective review of surgical outcomes is necessary when a sufficient number of cases are recruited in the near future.

\section{CONCLUSION}

We reported the first case of DILSPDP, and our findings indicate that it is a safe and feasible technique for benign or borderline malignant tumors in the body or tail of the pancreas.

\section{CONFLICTS OF INTEREST}

No potential conflict of interest relevant to this article was reported.

\section{REFERENCES}

1. Melotti G, Butturini G, Piccoli M, Casetti L, Bassi C, Mullineris B, et al. Laparoscopic distal pancreatectomy: results on a consecutive series of 58 patients. Ann Surg 2007:246:77-82

2. Yoon YS, Lee KH, Han HS, Cho JY, Ahn KS. Patency of splenic vessels after laparoscopic spleen and splenic vessel-preserving distal pancreatectomy. Br J Surg 2009;96:633-40.

3. Lee SE, Jang JY, Lee KU, Kim SW. Clinical comparison of distal pancreatectomy with or without splenectomy. J Korean Med Sci 2008:23:1011-4.

4. Hong TH, Lee SK, You YK, Kim JG. Singleport laparoscopic partial splenectomy: a case report. Surg Laparosc Endosc Percutan Tech 2010;20:e164-6.

5. Teh SH, Tseng D, Sheppard BC. Laparoscopic and open distal pancreatic resection for benign pancreatic disease. J Gastrointest Surg 2007;11:1120-5.

6. Shoup M, Brennan MF, McWhite K, Leung DH, Klimstra D, Conlon KC. The value of splenic preservation with distal pancreatectomy. Arch Surg 2002;137:164-8.

7. Lirici MM. Single site laparoscopic surgery: an intermediate step toward no (visible) scar surgery or the next gold standard in minimally invasive surgery? Minim Invasive Ther Allied Technol 2012;21:1-7.
8. Karakus SC, Kilincaslan H, Koku N, Ertaskin I. Is single port incisionless-intracorporeal conventional equipment-endoscopic surgery feasible in patients with retrocecal acute appendicitis? J Korean Surg Soc 2013;85:80-3.

9. Jung GO, Park DE, Chae KM. Clinical results between single incision laparoscopic cholecystectomy and conventional 3-port laparoscopic cholecystectomy: prospective case-matched analysis in single institution. J Korean Surg Soc 2012;83:374-80.

10. Chang SK, Lomanto D, Mayasari M. Single-port laparoscopic spleen preserving distal pancreatectomy. Minim Invasive Surg 2012;2012:197429. 